\title{
A new parameterization of particle dry deposition over rough surfaces
}

\author{
J. Zhang ${ }^{1,2}$ and Y. Shao ${ }^{2}$ \\ ${ }^{1}$ Key Laboratory of Mechanics on Disaster and Environment in Western China of the Ministry of Education of China, \\ Lanzhou University, 730000 Lanzhou, China \\ ${ }^{2}$ Institute for Geophysics and Meteorology, University of Cologne, 50937 Cologne, Germany
}

Correspondence to: J. Zhang (zhang-j@lzu.edu.cn)

Received: 6 January 2014 - Published in Atmos. Chem. Phys. Discuss.: 25 March 2014

Revised: 16 September 2014 - Accepted: 17 October 2014 - Published: 27 November 2014

\begin{abstract}
In existing particle dry deposition schemes, the effects of gravity and surface roughness elements on particle motion are often poorly represented. In this study, we propose a new scheme to overcome such deficiencies. Particle deposition velocity is a function of aerodynamic, surfacecollection and gravitational resistances. In this study, the effect of gravitation settling is treated analytically. More importantly, the new scheme takes into consideration the impacts of roughness elements on turbulent particle diffusion and surface particle collection. A relationship between aerodynamic and surface-collection processes is established by using an analogy between drag partition and depositionflux partition. The scheme is then tested against a windtunnel data set for four different surfaces and a good agreement between the scheme predictions and the observations is found. The sensitivity of the scheme to the input parameters is tested. Important factors which affect particle deposition in different particle size ranges are identified. The scheme shows good capacity for modeling particle deposition over rough surfaces.
\end{abstract}

\section{Introduction}

Particle dry deposition, the removal of particles from the atmosphere to the surface in absence of precipitation (Seinfeld and Pandis, 2006), can be divided into several sub-processes, including turbulence diffusion, surface collection and gravitational settling (Droppo, 2006). The inferential method is widely used to estimate particle deposition flux in terms of particle concentration and deposition velocity (or its in- verse, the resistance) (Sehmel, 1980; Slinn, 1982; Hicks et al., 1987; Wesely and Hicks, 2000; Raupach et al., 2001; Zhang et al., 2001; Petroff and Zhang, 2010; Seinfeld and Pandis, 2006; Kouznetsov and Sofiev, 2012). The effects of the sub-processes are represented with the corresponding resistances, i.e., turbulence diffusion, surface collection and gravitational settling are respectively related to the aerodynamic resistance, surface collection resistance and gravitational resistance. Deposition velocity, defined as the ratio of particle deposition flux and particle concentration is a quantity which describes the joint effect of the above mentioned resistances.

The usual approach to estimating deposition velocity is in analogy to electrical circuits: deposition velocity is considered to be the inverse of the deposition resistance which comprises of the contributions of the aerodynamic and surface collection resistances in series and the gravitational resistance in parallel (Hicks et al., 1987; Seinfeld and Pandis, 2006). Slinn (1982) deduced an analytical expression for particle deposition velocity over canopy surface based on the particle concentration equation. In his approach, the gravitational effect was not considered at first, but then later added to the result.

The existing deposition-velocity approach has two deficits. Firstly, while the gravitational resistance is often treated as a resistance in parallel to the turbulent diffusion resistance, gravitational settling is not driven by concentration gradient and the settling process cannot be expressed in an electricalcircuit analogy. More specifically, the usual treatment of gravitational settling as a parallel resistance (Slinn, 1982; Zhang et al., 2001; Petroff and Zhang, 2010), including the 

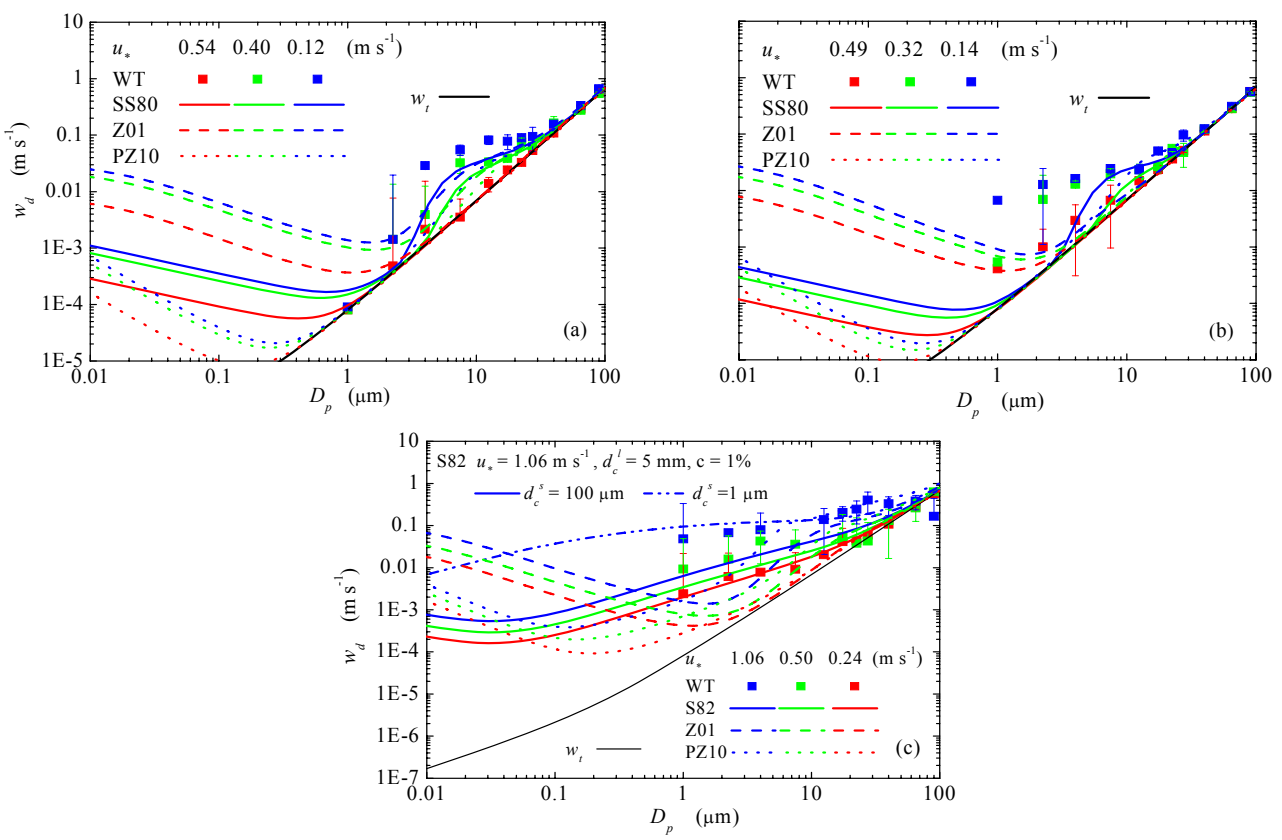

Figure 1. Comparison of deposition velocity predicted by the SS80, S82, Z01 and PZ10 schemes (lines) with the wind-tunnel measurements (dots) over three different surfaces. (a) Sticky wood; (b) Sand; (c) Plant. The wind field parameters of the schemes are consistent with the relevant wind-tunnel experiments.

modified version of Seinfeld and Pandis (2006), does not satisfy the particle mass conservation requirement (Venkatram and Pleim, 1999). Secondly, the collection of particles by the surface is normally described based on the studies of particle deposition on isolated collector (Petroff et al., 2008). Kouznetsov and Sofiev (2012) reported a more detailed scheme for particle dry deposition, but the parameter of "collection scale" they introduced does not have a clear physical interpretation and is thus practically difficult to determine. In particle deposition schemes, the typical size of the surface collectors is often the only parameter used to for the characterization of the surface, which is insufficient for rough surfaces.

The deficiencies of the existing particle deposition schemes are clearly revealed in our recent comparison of the Slinn and Slinn (1980, SS80 hereafter), Slinn (1982, S82 hereafter), Zhang et al. (2001, Z01 hereafter) and Petroff and Zhang (2010, PZ10 hereafter) with the wind-tunnel observations, as described in the companion paper by Zhang et al. (2014). The results of the latter study are summarized in Fig. 1 which shows that the performances of existing deposition schemes are unsatisfactory, especially for rough surfaces (e.g., surface with plants). Considering that the Z01 and PZ10 schemes are based on field observation data sets and their model coefficients may have included the effects of certain factors (such as atmospheric convection and complex surface conditions) which cannot be well simulated in our wind-tunnel experiments, we will use SS80 and S82 as reference schemes in this study. As shown in Fig. 1c, the model-observation discrepancies can be reduced by tuning some input parameters of S82, but the tuned parameters are physically unrealistic.

In this paper, a new parameterization of particle dry deposition is proposed. The deposition velocity is derived from the particle concentration equation with a boundary condition which involves the surface-collection process. The relationship between surface momentum flux (drag) and deposition flux is established by combining momentum depletion and particle collection. The drag partition theory and its parameterization are introduced to describe the surfacecollection process in the new scheme. The effects of gravitational settling and surface collectors over a rough surface are now adequately dealt with. Finally, the new scheme is validated by the measurements of the wind-tunnel experiments as described in Zhang et al. (2014).

\section{Parameterization scheme for particle deposition}

\subsection{Assumptions}

We firstly introduce the assumptions for the new scheme. Following Raupach (1992) and Shao and Yang (2005, 2008), we consider a rough surface to be a flat ground surface superposed with roughness elements (rocks, trees, buildings etc.) as illustrated in Fig. 2a. The roughness elements are assumed to be uniform in size and randomly distributed on the surface (Fig. 2b). The flow and particle fields over the surface are in steady state and horizontally homogeneous. 


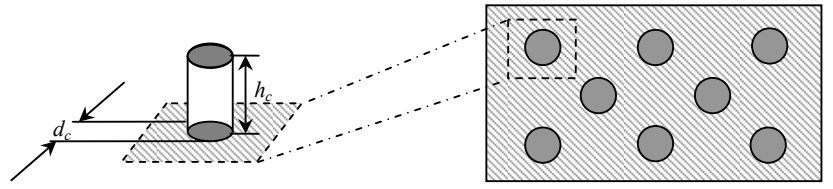

(a) Roughness element

(b) Top view of rough surface

Figure 2. Illustration of rough surface. (a) A roughness element with height $h_{\mathrm{c}}$ and diameter $d_{\mathrm{c}}$; (b) Roughness elements randomly distributed on the surface.

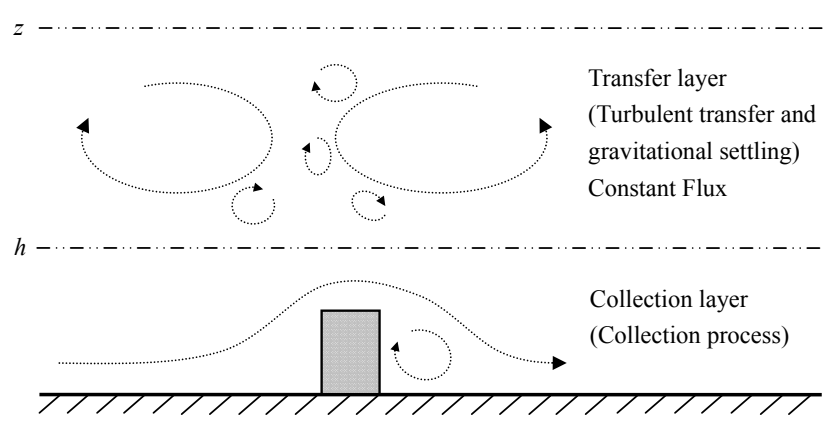

Figure 3. Illustration of the two-layer model. The lower layer, from the ground to the top of the laminar (or quasi-laminar) layer, is the collection layer where the particle collection process takes place. Over the collection layer is the transfer layer, where turbulent transfer and gravitational settling are dominant and the particle flux is vertically constant. Air flow is represented by the dash lines.

The atmospheric boundary layer is divided into two parts (Fig. 3). The upper part is the transfer layer, in which particle is transported mainly by eddy diffusion and gravitation settling. As particle concentration is in steady state and horizontally homogeneous, the particle deposition flux, $F_{\mathrm{d}}$, is vertically constant and obeys the following equation (Shao, 2008):

$F_{\mathrm{d}}=-\left(k_{\mathrm{p}}+K_{\mathrm{p}}\right) \cdot \frac{\partial c}{\partial z}-w_{\mathrm{t}} \cdot c$,

where $c$ is particle concentration, $k_{\mathrm{p}}$ particle Brownian diffusivity, $K_{\mathrm{p}}$ particle eddy diffusivity, and $w_{\mathrm{t}}$ the particle terminal velocity. $F_{\mathrm{d}}$ is upward positive.

The lower part under the transfer layer is the collection layer with thickness of

$h=h_{\mathrm{c}}+\delta$,

where $h_{\mathrm{c}}$ is the roughness element height and $\delta$ the thickness of the laminar layer over the roughness elements. The laminar layer may be broken at the top of the roughness elements and $h_{\mathrm{c}}$ is usually much larger than $\delta$. Therefore, in general, the thickness of the collection layer is $h_{\mathrm{c}}$ for a rough surface and $\delta$ for a smooth surface.

Since $F_{\mathrm{d}}$ is vertically constant and deposition velocity is defined as $w_{\mathrm{d}}=-F_{\mathrm{d}} / c\left(w_{\mathrm{d}}\right.$ is downward positive). By solv- ing Eq. (1), one obtains that

$w_{\mathrm{d}}(z)=\left(r_{\mathrm{g}}+\frac{r_{\mathrm{s}}-r_{\mathrm{g}}}{\exp \left(r_{\mathrm{a}} / r_{\mathrm{g}}\right)}\right)^{-1}$,

with the boundary condition

$w_{\mathrm{d}}(h)=-\frac{F_{\mathrm{d}}}{c(h)}=\frac{1}{r_{\mathrm{s}}}$,

where $r_{\mathrm{a}}$ is the aerodynamic resistance accounting for the particle diffusion, given by

$r_{\mathrm{a}}(z)=\int_{h}^{z} \frac{1}{K_{\mathrm{p}}(z)+k_{\mathrm{p}}} d z$.

$r_{\mathrm{s}}$ is the surface collection resistance, and $r_{\mathrm{g}}$ the gravitational resistance defined as the inverse of particle terminal velocity, i.e., $r_{\mathrm{g}}=w_{\mathrm{t}}^{-1}$.

Here, the expression of $w_{\mathrm{d}}$ (i.e., Eq. 3, an analytical solution of Eq. 1) is not based on electrical-circuit analogy and $r_{\mathrm{g}}$ is no longer treated as a parallel resistance. So the first deficiency we described in the introduction has been dealt with.

\subsection{Aerodynamic resistance}

In the transfer layer, $k_{\mathrm{p}}$ is much smaller than $K_{\mathrm{p}}$ and thus negligible. $K_{\mathrm{p}}$ can be derived from eddy viscosity $K_{\mathrm{T}}$. Csanady (1963) derived an expression of the ratio of $K_{\mathrm{T}} / K_{\mathrm{p}}$ (i.e., $S c_{\mathrm{T}}$, the turbulent Schmidt number) by taking the trajectory-crossing effect into consideration

$S c_{\mathrm{T}}=\frac{K_{\mathrm{T}}}{K_{\mathrm{p}}}=\left(1+\frac{\alpha^{2} w_{\mathrm{t}}^{2}}{\sigma^{2}}\right)^{1 / 2}$,

where $\alpha$ is a dimensionless coefficient and $\sigma$ the standard deviation of the turbulent velocity. In this study, $\alpha$ is taken as 1 and $\sigma$ as friction velocity $u_{*}$. The expression of $K_{\mathrm{T}}$ is normally found as (Seinfeld and Pandis, 2006)

$K_{\mathrm{T}}=\frac{\kappa u_{*}\left(z-z_{\mathrm{d}}\right)}{\varphi(\varsigma)}$

where $\kappa$ is the von Karman constant, and $z_{\mathrm{d}}$ the zero-plane displacement height, $\varphi$ a stability function, $\zeta=\left(z-z_{\mathrm{d}}\right) / L$ and $L$ the Obukhov length.

An integration of Eq. (5) yields

$r_{\mathrm{a}}(z)=\frac{S c_{\mathrm{T}}}{\kappa u_{*}}\left\{\left[\varphi(\varsigma) \cdot \ln \left(z-z_{\mathrm{d}}\right)\right]_{h}^{z}-\int_{h}^{z} \ln \left(z-z_{\mathrm{d}}\right) d(\varphi)\right\}$.

For neutral atmospheric boundary layers, $\varphi=1$. Then we have

$r_{\mathrm{a}}(z)=\frac{S c_{\mathrm{T}}}{\kappa u_{*}} \ln \left(\frac{z-z_{\mathrm{d}}}{h_{\mathrm{c}}-z_{\mathrm{d}}}\right)$ for rough surfaces,

$r_{\mathrm{a}}(z)=\frac{B_{1} \cdot S c_{\mathrm{T}}}{\kappa u_{*}} \ln \left(\frac{z}{z_{0}}\right)$ for smooth surfaces,

where $B_{1}$ is an empirical constant determined by the airflow characteristic over the surface. The term $B_{1} \cdot S c_{\mathrm{T}}$ is set to 
0.6 for SS80 and 1 for Zhang et al. (2001). In this study, the value of $B_{1}$ is estimated to be 0.45 , based on the wind-tunnel measurements of Zhang et al. (2014).

\subsection{Gravitational resistance}

In the Stokes regime, $r_{\mathrm{g}}$ can be calculated as

$r_{\mathrm{g}}=\left(T_{\mathrm{p}} \cdot g\right)^{-1}$,

where $g$ is the gravitational acceleration and

$$
T_{\mathrm{p}}=\frac{C_{\mathrm{c}} \rho_{\mathrm{p}} D_{\mathrm{p}}^{2}}{18 \mu}
$$

is the particle relaxation time. $C_{\mathrm{c}}$ the Cunningham correction factor which accounts for non-continuum effects when calculating drag on small particles, $D_{\mathrm{p}}$ particle diameter, $\rho_{\mathrm{p}}$ particle density and $\mu$ air viscosity.

\subsection{Surface collection resistance}

The surface collection resistance is the essence of the lower boundary condition for solving Eq. (1), which is given either in form of the deposition flux or particle concentration at the surface. As the rough surface is considered to be a smooth surface superposed with roughness elements (Fig. 2), it comprises upward facing areas (ground and roof of roughness element) and the side areas of the roughness elements. The deposition flux can be thus partitioned to several components which correspond to the deposition fluxes to these areas, similar to drag partition. By doing so, a relationship between the particle flux partition and drag partition can be established and the drag partition theory enables the estimation of the surface collection resistance.

In analogy to drag partition theory (e.g., Arya, 1975; Raupach, 1992; Shao and Yang, 2005, 2008), the deposition flux can be split into three parts:

$F_{\mathrm{d}}=F_{\mathrm{d}, \mathrm{c}}+F_{\mathrm{d}, \mathrm{s}}+F_{\mathrm{d}, \mathrm{r}}$,

where $F_{\mathrm{d}, \mathrm{c}}$ is the particle flux due to particle collection by the roughness elements (collectors), $F_{\mathrm{d}, \mathrm{s}}$ is that deposited on the ground surface and $F_{\mathrm{d}, \mathrm{r}}$ on the roof of the roughness elements.

Per definition, the force exerted on roughness elements (pressure drag) can be calculated as

$\tau_{\mathrm{c}}=C_{\mathrm{d}} \cdot\left[\rho_{\mathrm{a}}\left|u_{\mathrm{a}}(h)\right| \cdot u_{\mathrm{a}}(h)\right] \cdot \lambda$,

where $C_{\mathrm{d}}$, the drag coefficient for isolated roughness element, is approximately 0.3 (Shao and Yang, 2008), $\rho_{\mathrm{a}}$ air density, $\lambda$ the frontal area index $\left(\sim d_{\mathrm{c}} h_{\mathrm{c}}\right)$ of the roughness elements and $u_{\mathrm{a}}$ the air horizontal speed. Similarly, the particle flux due to particle collection by the roughness elements can be expressed as

$F_{\mathrm{d}, \mathrm{c}}=-E \cdot\left|u_{\mathrm{a}}(h)\right| \cdot c(h) \cdot \lambda$, where $c$ is particle concentration and $E$ particle collection efficiency of isolated roughness element.

A combination of Eqs. (13) and (14) yields the relationship between the pressure drag and the deposited flux due to roughness element collection and thus the expression of $F_{\mathrm{d}, \mathrm{c}}$ can be written as

$F_{\mathrm{d}, \mathrm{c}}=-\frac{\tau_{\mathrm{c}}}{\tau} \cdot \frac{\tau}{\rho_{\mathrm{a}} u_{\mathrm{a}}(h)} \cdot \frac{E}{C_{\mathrm{d}}} \cdot c(h)$,

where $\tau$ is the total shear stress (or drag) on the surface. The element collection efficiency, $E$, represents the collected fraction of all particles initially moving on a collision course with the roughness elements. It consists of the contributions of Brownian motion, impaction and interception, i.e.,

$E=E^{\mathrm{B}}+E^{\mathrm{im}}+E^{\mathrm{in}}$,

where $E^{\mathrm{B}}$ is the collection efficiency caused by Brownian motion and can be estimated following Petroff et al. (2008):

$E^{\mathrm{B}}=C_{\mathrm{B}} S c^{-2 / 3} R e^{n_{\mathrm{B}}-1}$,

where $S c=v / k_{\mathrm{p}}$ is the Schmidt number with $v$ being the air kinematic viscosity and $k_{\mathrm{p}}$ the particle molecular diffusivity. $R e$ is the roughness element Reynolds number. $C_{\mathrm{B}}$ and $n_{\mathrm{B}}$ are parameters depending on flow regimes as shown in Table 1.

$E^{\mathrm{im}}$ is the impaction efficiency due to particle collection on roughness element. Following Petroff et al. (2008), we have,

$E^{\mathrm{im}}=\left(\frac{S t}{0.6+S t}\right)^{2}$,

where $S t=T_{\mathrm{p}} u_{*} / d_{\mathrm{c}}$.

Taking into account of the possible particle growth, $D_{\mathrm{p}, \delta}$ is used to distinguish from $D_{\mathrm{p}}$ for describing the size of grown particles moving close to wet surface. $D_{\mathrm{p}, \delta}$ can be estimated following Fitzgerald (1975) or Gerber (1985). Later, the subscript $\delta$ is introduced (e.g., $T_{\mathrm{p}, \delta}$ ) to describe the replacement of $D_{\mathrm{p}}$ with $D_{\mathrm{p}, \delta}$ in the relevant calculations.

$E^{\text {in }}$ is the collection efficiency due to interception. Based on the theoretical results for potential flows, Fuchs (1964) suggested that $E^{\text {in }}$ should be directly proportional to particle size $\left(D_{\mathrm{p}}\right)$ and inversely proportional to the size of roughness element $\left(d_{\mathrm{c}}\right)$. Slinn (1982) considered that in addition to the size of the roughness element, the micro roughness characteristics (i.e., the characteristics of the roughness element surface, e.g., hair on plant leaves) are also important for interception. Our wind-tunnel study (Zhang et al., 2014) shows $E^{\text {in }}$ is also enhanced by friction velocity, $u_{*}$. In summary, it is appropriate to propose that

$E^{\text {in }}=A_{\text {in }} \cdot u_{*} \cdot 10^{-S t} \cdot \frac{2 D_{\mathrm{p}, \delta}}{d_{\mathrm{c}}}$.

According to the definition, interception describes the behaviors of particles which can follow the airflow well. The term 
Table 1. Typical values of $C_{\mathrm{B}}$ and $n_{\mathrm{B}}$ in Eq. (17) for different Reynolds numbers; Petroff et al. (2008).

\begin{tabular}{lll}
\hline$R e$ & $C_{\mathrm{B}}$ & $n_{\mathrm{B}}$ \\
\hline $1-4 \times 10^{3}$ & 0.467 & $1 / 2$ \\
$4 \times 10^{3}-4 \times 10^{4}$ & 0.203 & $3 / 5$ \\
$4 \times 10^{4}-4 \times 10^{5}$ & 0.025 & $4 / 5$ \\
\hline
\end{tabular}

$10^{-S t}$ is introduced to correct the deviation from this requirement, and it approaches 1 for particles of small inertial. To account for the effect of micro-roughness characteristics, the term $A_{\text {in }} u_{*}$ is introduced, with $A_{\text {in }}$ being an empirical parameter related to the micro-roughness characteristics, e.g., the ratio of hair size to roughness element size.

Particle deposition to the roof of roughness element and the ground surfaces is caused by the mechanisms of gravitational settling, and Brownian diffusion and impaction; thus we have

$F_{\mathrm{d}, \mathrm{r}}=F_{\mathrm{d}, \mathrm{r}}^{\mathrm{g}}+F_{\mathrm{d}, \mathrm{r}}^{\mathrm{B}}+F_{\mathrm{d}, \mathrm{r}}^{\mathrm{im}}$,

$F_{\mathrm{d}, \mathrm{s}}=F_{\mathrm{d}, \mathrm{s}}^{\mathrm{g}}+F_{\mathrm{d}, \mathrm{s}}^{\mathrm{B}}+F_{\mathrm{d}, \mathrm{s}}^{\mathrm{im}}$,

where $F_{\mathrm{d}, \mathrm{r}}^{\mathrm{g}}$ and $F_{\mathrm{d}, \mathrm{s}}^{\mathrm{g}}$ are caused by gravitational settling, $F_{\mathrm{d}, \mathrm{r}}^{\mathrm{B}}$ and $F_{\mathrm{d}, \mathrm{s}}^{\mathrm{B}}$ by Brownian diffusion and $F_{\mathrm{d}, \mathrm{r}}^{\mathrm{im}}$ and $F_{\mathrm{d}, \mathrm{s}}^{\mathrm{im}}$ by impaction.

The gravitational settling fluxes can be calculated as

$F_{\mathrm{d}, \mathrm{r}}^{\mathrm{g}}=-w_{\mathrm{t}, \delta} \cdot c(h) \cdot \eta$,

$F_{\mathrm{d}, \mathrm{s}}^{\mathrm{g}}=-w_{\mathrm{t}, \delta} \cdot c(h) \cdot(1-\eta)$,

where $\eta$ is the basal area index (fraction of cover) of the roughness elements. The terminal velocity of particles near the surface, $w_{\mathrm{t}, \delta}$, is calculated as

$w_{\mathrm{t}, \delta}=T_{\mathrm{p}, \delta} \cdot g$.

Brownian diffusion is another important mechanism responsible for particle (especially very small particles) to move across the laminar layer. This process of particle transfer is closely related to momentum transfer. Particles, for which Brownian diffusion is effective, usually do not rebound from the surface (Chamberlain, 1967). For these particles, the surface particle concentration, $c(0)$, can be assumed to be zero. We therefore have

$F_{\mathrm{d}, \mathrm{r}}^{\mathrm{B}}=-k_{\mathrm{p}} \cdot \frac{c(h)}{\delta} \cdot \eta$,

$\tau_{\mathrm{r}}=v \cdot \frac{\rho_{\mathrm{a}} u_{\mathrm{a}}(h)}{\delta} \cdot \eta$.

A combination of Eqs. (23a) and (23b) leads to

$F_{\mathrm{d}, \mathrm{r}}^{\mathrm{B}}=-\frac{\tau_{\mathrm{r}}}{\rho_{\mathrm{a}} u_{\mathrm{a}}(h)} \cdot S c^{-1} \cdot c(h)$.
According to the drag partition theory, the drag on the ground surface is

$\tau_{\mathrm{s}}=\tau-\tau_{\mathrm{c}}-\tau_{\mathrm{r}}$

where $\tau$ is the total shear stress (or drag) on the surface, $\tau_{\mathrm{c}}$ the pressure drag and $\tau_{\mathrm{r}}$ the drag on the roof of the roughness elements. The pressure drag, $\tau_{\mathrm{c}}$, leads to a momentum reduction of the mean flow by production of turbulence, and the enhanced turbulence has a positive contribution to the Brownian diffusion over the ground surface. Further, we assume $c(\delta)=c(h)$. In analogy to Eq. (24), the deposition flux caused by Brownian diffusion to the ground surface is

$F_{\mathrm{d}, \mathrm{s}}^{\mathrm{B}}=-\frac{\tau+\tau_{\mathrm{c}}-\tau_{\mathrm{r}}}{\rho_{\mathrm{a}} \cdot u_{\mathrm{a}}(h)} \cdot S c^{-1} \cdot c(h)$.

Particle is also collected by the surfaces due to turbulent impaction. Studies show that turbulent impaction is dependent on turbulence near the surface and the dimensionless particle relaxation time $T_{\mathrm{p}, \delta}^{+}$. Following SS80, particle deposition due to impaction on an upward facing surface can be expressed as

$F_{\mathrm{d}, \mathrm{r}}^{\mathrm{im}}+F_{\mathrm{d}, \mathrm{s}}^{\mathrm{im}}=-\frac{\tau}{\rho_{\mathrm{a}} \cdot u_{\mathrm{a}}(h)} \cdot 10^{-\frac{3}{T_{\mathrm{p}, \delta}^{+}}} \cdot c(h)$,

where $T_{\mathrm{p}, \delta}^{+}$is defined as

$T_{\mathrm{p}, \delta}^{+}=\frac{T_{\mathrm{p}, \delta} \cdot u_{*}^{2}}{v}$.

Finally, it follows from Eqs. (12) to (28) that

$$
\begin{aligned}
F_{\mathrm{d}}= & -\left\{\frac { \tau } { \rho _ { \mathrm { a } } \cdot u _ { \mathrm { a } } ( h ) } \left[\frac{E}{C_{\mathrm{d}}} \cdot \frac{\tau_{\mathrm{c}}}{\tau}+\left(1+\frac{\tau_{\mathrm{c}}}{\tau}\right)\right.\right. \\
& \left.\left.\cdot S c^{-1}+10^{-\frac{3}{T_{\mathrm{p}, \delta}^{+}}}\right]+w_{\mathrm{t}, \delta}\right\} c(h) .
\end{aligned}
$$

According to Eq. (4) and taking account of the rebound effect, the surface collection resistance is found to be

$r_{\mathrm{s}}=\left\{R \cdot w_{\mathrm{dm}}\left[\frac{E}{C_{\mathrm{d}}} \cdot \frac{\tau_{\mathrm{c}}}{\tau}+\left(1+\frac{\tau_{\mathrm{c}}}{\tau}\right) \cdot S c^{-1}+10^{-\frac{3}{T_{\mathrm{p}, \delta}^{+}}}\right]+w_{\mathrm{t}, \delta}\right\}^{-1}$,

where

$R=\exp (-b \sqrt{S t})$,

with $b$ being an empirical constant of about 2 (Chamberlain, 1967). In the studies of Giorgi (1988) and Zhang et al. (2001), $b$ is set to 1. In Eq. (30),

$w_{\mathrm{dm}}=\frac{\tau}{\rho_{\mathrm{a}} \cdot u_{\mathrm{a}}\left(h_{\mathrm{c}}\right)}$,

is the conductance for momentum. For smooth surfaces, $w_{\mathrm{dm}}$ is given by

$w_{\mathrm{dm}}=B_{2} \cdot u_{*}$, 
Table 2. Summary of the new particle deposition scheme and the scheme input parameters. According to the drag partition theory, $z_{0}$ and $z_{\mathrm{d}}$ which are not considered as input parameters can be estimated from surface parameters and $u_{*}$ (Shao and Yang, 2005, 2008). The particle density is considered as a constant $\left(2200 \mathrm{~kg} \mathrm{~m}^{-3}\right)$ in this study.

\begin{tabular}{|c|c|c|c|c|c|c|}
\hline \multicolumn{7}{|c|}{ Parameterization } \\
\hline Wind field & \multicolumn{5}{|c|}{ Surface } & Particle \\
\hline$u_{*}$ & $h_{\mathrm{c}}$ & $d_{\mathrm{c}}$ & $\lambda$ & $A_{\text {in }}$ & $b$ & $D_{\mathrm{p}}$ \\
\hline \multicolumn{7}{|c|}{ Rough surface } \\
\hline$r_{\mathrm{a}}(z)=\frac{S c_{\mathrm{T}}}{\kappa \cdot u_{*}} \ln \left(\frac{z-z_{\mathrm{d}}}{h_{\mathrm{c}}-z_{\mathrm{d}}}\right)$ & $w_{\mathrm{dm}}=\frac{u_{*}}{u_{\mathrm{a}} h_{\mathrm{c}}}$ & $\frac{\tau_{\mathrm{c}}}{\tau}=f(n, \lambda, \eta)$ & $E=E^{\mathrm{B}}+E^{\mathrm{im}}+E^{\mathrm{in}}$ & $D_{\mathrm{p}, \delta}$ & $R$ & $r_{\mathrm{g}}=\frac{18 \mu}{C_{\mathrm{c}} \rho_{\mathrm{p}} D_{\mathrm{p}}^{2} g}$ \\
\hline \multicolumn{7}{|c|}{ Smooth surface } \\
\hline$r_{\mathrm{a}}(z)=\frac{B_{1} \cdot S_{c_{\mathrm{T}}}}{\kappa \cdot u_{*}} \cdot \ln \left(\frac{z}{z_{0}}\right)$ & $w_{\mathrm{dm}}=B_{2} u_{*}$ & \multirow[t]{2}{*}{ drag partition } & \multirow[t]{2}{*}{ element collection } & \multirow[t]{2}{*}{ particle growth } & \multirow[t]{2}{*}{ rebound } & \multirow[t]{2}{*}{ settling resistance } \\
\hline$B_{1}=0.45$ & $B_{2}=3$ & & & & & \\
\hline & $r_{\mathrm{s}}=\{$ & $R \cdot w_{\mathrm{dm}}\left[\frac{E}{C_{\mathrm{d}}} \cdot \frac{\tau_{\mathrm{c}}}{\tau}+\right.$ & $\left.1+\frac{\tau_{\mathrm{c}}}{\tau}\right) \cdot S c^{-1}+10^{-\frac{3}{T_{\mathrm{p}, \delta}^{+}}}$ & ]$\left.+w_{\mathrm{t}, \delta}\right\}^{-1}$ & & \\
\hline \multicolumn{7}{|c|}{$w_{\mathrm{d}}(z)=\left(r_{\mathrm{g}}+\frac{r_{\mathrm{s}}-r_{\mathrm{g}}}{\exp \left(r_{\mathrm{a}} / r_{\mathrm{g}}\right)}\right)^{-1}$} \\
\hline
\end{tabular}

where $B_{2}$ is an empirical constant of about 3 (Zhang et al., 2001).

The term $\tau_{\mathrm{c}} / \tau$ can be evaluated following the drag partition formulation of Shao and Yang (2005):

$\frac{\tau_{\mathrm{c}}}{\tau}=\frac{\beta \lambda_{\mathrm{e}}}{1+\beta \lambda_{\mathrm{e}}}$

and

$\lambda_{\mathrm{e}}=\frac{\lambda}{(1-\eta)^{c_{2}}} \cdot \exp \left(-\frac{c_{1} \lambda}{(1-\eta)^{c_{2}}}\right)$,

with $c_{1}=6, c_{2}=0.1$. $\beta$ is the ratio of the pressure-drag coefficient to friction-drag coefficient, which varies with surface type. Here, we set $\beta$ to 200 following the study of Shao and Yang (2005).

To sum up, the parameters used in the new scheme are organized and shown in Table 2. In comparison with the existing particle deposition schemes, the new scheme appears to require three additional parameters for the characterization of the rough surface, namely, $h_{\mathrm{c}}, \lambda$ and $\eta$ (or $d_{\mathrm{c}}$ ). If the aspect ratio of the roughness elements is given, then only two additional parameters $\left(h_{\mathrm{c}}\right.$ and $\left.\lambda\right)$ are necessary. Note however $z_{\mathrm{d}}$ and $z_{0}$ used for wind profile description can be expressed following Shao and Yang (2008) in terms of $h_{\mathrm{c}}, \lambda$ and $\eta$. Thus, the new scheme requires only one parameter more than existing schemes. If $h_{\mathrm{c}} / d_{\mathrm{c}}$ is specified, then, it requires no more parameters than the existing schemes.

\section{Validation}

For validation, we test the new scheme for four different surfaces studied in the wind-tunnel experiment of Zhang et al. (2014). The values of relevant parameters are listed in Table 3. The predictions of deposition velocity as a function of particle size are compared with the wind-tunnel measurements and the results of the SS80 and/or S82 schemes (Fig. 4).

For the sticky wood surface, roughness elements are absent. Particle collection is realized through impaction, Brownian motion and gravitational settling. Particle rebound should be very low for the stickiness of the surface. As shown in Fig. 4a, the new scheme well predicted the wind-tunnel observations and performed better than the SS80 scheme. It should be pointed out that due to the limitations of the measurement device (the PDA: particle dynamics analysis) used in the wind-tunnel experiments comparison can only be made for particles bigger than $1 \mu \mathrm{m}$.

The sand surface is used as the second case to test the new scheme (Fig. 4b). The difference between the sand surface and the sticky wood surface is the presence of the sand particles (act as roughness elements, although their sizes are small) not only enhances turbulence over the surface but also improves the surface collection efficiency. In our scheme, the size of the elements is taken to be the average diameter of the sand particles $\left(D_{\text {sand }}=0.2 \mathrm{~mm}\right.$ ) and the element height half that diameter (i.e., $d_{\mathrm{c}}=0.2 \mathrm{~mm}, h_{\mathrm{c}}=0.1 \mathrm{~mm}$ ). The sand particles are assumed to be distributed uniformly on the surface and the distance between them twice the diameter. The other surface parameters, such as the frontal area and basal area indices can be calculated according to these assumptions. The rebound effect is taken into account and the parameter $b$ is set to 1 . For smooth sand grains (no hair), $A_{\text {in }}$ is set to 1 . 
Table 3. Parameters for validation of the new scheme for the four different surfaces studied in the wind-tunnel experiments of Zhang et al. (2014). For all tests, particle density $\rho_{\mathrm{p}}=2200 \mathrm{~kg} \mathrm{~m}^{-3}$ is used. The wind parameters are obtained from the experimental data.

\begin{tabular}{llllllllll}
\hline & $z_{\mathrm{r}}(\mathrm{mm})$ & $u_{*}\left(\mathrm{~m} \mathrm{~s}^{-1}\right)$ & $z_{0}(\mathrm{~mm})$ & $z_{\mathrm{d}}(\mathrm{mm})$ & $h_{\mathrm{c}}(\mathrm{mm})$ & $d_{\mathrm{c}}(\mathrm{mm})$ & $\lambda$ & $A_{\text {in }}$ & $b$ \\
\hline Sticky wood & 15 & 0.12 & 0.075 & 0 & 0 & 0 & 0 & 1 & 0 \\
& & 0.40 & 0.033 & 0 & 0 & 0 & 0 & 1 & 0 \\
& & 0.54 & 0.032 & 0 & 0 & 0 & 0 & 1 & 0 \\
\hline \multirow{2}{*}{ Sand } & 15 & 0.14 & 0.153 & 0 & 0.1 & 0.2 & 0.125 & 1 & 1 \\
& & 0.32 & 0.143 & 0 & 0.1 & 0.2 & 0.125 & 1 & 1 \\
& & 0.49 & 0.135 & 0 & 0.1 & 0.2 & 0.125 & 1 & 1 \\
\hline \multirow{2}{*}{ Plant } & 250 & 0.24 & 5.927 & 200 & 230 & 5 & 0.4 & 150 & 0.01 \\
& & 0.50 & 2.877 & 200 & 230 & 5 & 0.4 & 150 & 0.01 \\
& & 1.06 & 2.106 & 200 & 230 & 5 & 0.4 & 150 & 0.01 \\
\hline \multirow{2}{*}{ Water } & 25 & 0.15 & 0.300 & 0 & $30 z_{0}$ & 0.1 & 0.538 & 100 & 0 \\
& & 0.36 & 0.306 & 0 & $30 z_{0}$ & 0.1 & 0.538 & 100 & 0 \\
& & 0.57 & 0.309 & 0 & $30 z_{0}$ & 0.1 & 0.538 & 100 & 0 \\
\hline
\end{tabular}
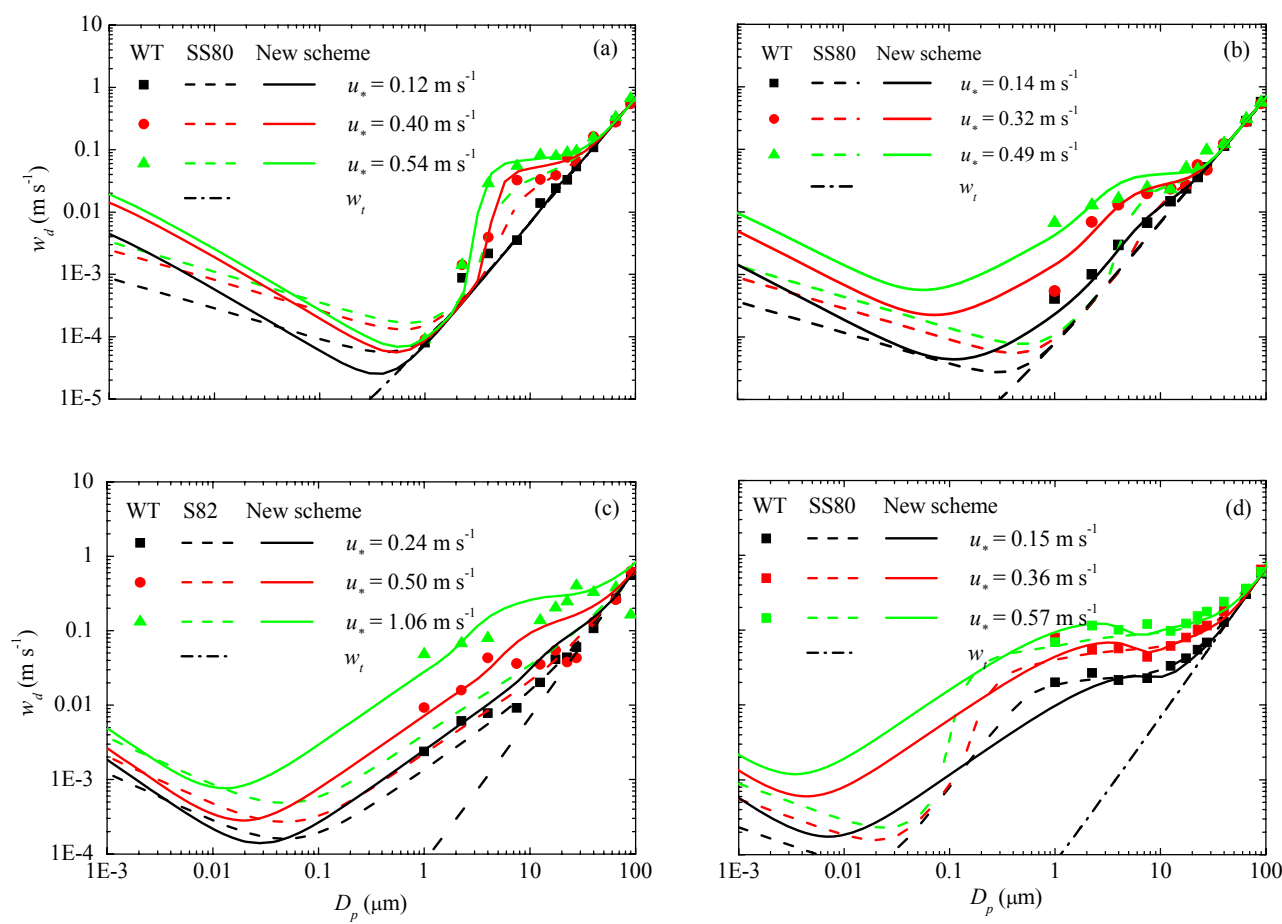

Figure 4. Comparison of deposition velocity, $w_{\mathrm{d}}$, as a function of particle diameter, $D_{\mathrm{p}}$, predicted by the new scheme (solid lines) and the SS80 or S82 scheme (dashed lines) with the wind-tunnel (WT) measurements (dots) for the (a) sticky wood, (b) sand, (c) plant and (d) water surface.

Again, the predictions of the new scheme agree well with the experimental data (Fig. 4b). Compared with the SS80 scheme, the new scheme is obviously an improvement, especially for the particle size range $1-10 \mu \mathrm{m}$. The enhancement of the deposition velocity can be attributed to the better treatment of interception (related to $d_{\mathrm{c}}$ ) in the new scheme, which is neglected in the SS80 scheme. The comparison shows that even small roughness elements on a surface can play an important role in the process of particle deposition.

The third case is the plant surface with rather complex structures. The roughness element (branch) size is $d_{\mathrm{c}}=$ $5 \mathrm{~mm}$ and the height $h_{\mathrm{c}}=230 \mathrm{~mm}$. Taking into account the effect of leaves, we set $A_{\text {in }}=150$ and $\lambda=0.4$. The predictions of the new scheme shown in Fig. 4c agree well with the experimental data and are better than the results of the S82 
scheme. Compared with the sand surface, the deposition velocity over plant surface is increased, which may be mainly caused by the enhanced friction velocity $u_{*}$.

We also tested the new scheme for the water surface. As shown in Fig. 4d, if particle size growth due to high humidity near the water surface is assumed $(\mathrm{RH}=100 \%)$, then the predicted deposition velocity with the SS80 scheme can be made to match the experimental data. However, this good agreement is for the wrong reason: the fused silica particles used in the experiments are not hygroscopic, to which the particle growth theory (Fitzgerald, 1975) does not apply. On the other hand, it is incorrect to treat the water surface under windy conditions as a smooth surface because of the bubbles and spray droplets caused by the hitting of the water waves on the wall of the trays used as water containers in the experiment.

The new scheme allows a better description of particle deposition on the water surface which under windy conditions can be treated as a rough surface with waves and bubbles acting as roughness elements. The input parameters used in the new scheme are taken as $h_{\mathrm{c}}=30 z_{0}, d_{\mathrm{c}}=0.1 \mathrm{~mm}$ and the distances between the adjacent elements are supposed to be equal to $h_{\mathrm{c}}$. The other surface parameters, including element density and frontal area index, can be computed from these parameters. Spray droplets over the water surface behave like hair on plant leaves, and we therefore set $A_{\text {in }}=100$. Using the wind field parameters derived from the wind-tunnel experiments, the deposition velocities for different particle sizes are calculated. The results shown in Fig. $4 d$ confirm the good agreement between the scheme predictions with the experimental data. We have shown that the enhanced deposition over the water surface is indeed not due to particle growth, but due to the enhanced collection capacities of the water surface caused by waves, bubbles and spray droplets.

\section{Sensitivity analysis}

The main advantage of the new scheme is the improved capacity for parameterization of particle deposition to rough surfaces and the results shown in the previous section highlighted this capacity through comparison with the windtunnel observations. As the scheme performance depends on the certainty of the input parameters listed in Table 2, it is important to examine the sensitivity of the scheme to these parameters and to identify the most influential ones.

Table 2 shows that particle deposition depends on particle properties (size and density), aerodynamic conditions (friction velocity, roughness length and zero-plane displacement) and surface characteristics (roughness element height, frontal and basal area indexes). These parameters are not all necessarily independent, because roughness length and zeroplane displacement are functions of the surface characteristics (Shao and Yang, 2005, 2008).
We first consider the sensitivity of particle deposition to particle properties. The typical behavior of the deposition velocity as a function of particle size is as shown in Fig. 4: deposition velocity is large for small particles $(<0.01 \mu \mathrm{m})$ because of Brownian diffusion and is large for big particles $(>50 \mu \mathrm{m})$ because of gravitational settling. Particle deposition is negligible for particles in the range from 0.01 to $50 \mu \mathrm{m}$, because they are too big for Brownian diffusion and too small for gravitational settling. Normally, the minimum deposition velocity occurs in the range from 0.1 to $1 \mu \mathrm{m}$ (Fig. 4a and b), but the enhancement of interception shifts this range to smaller particles (Fig. $4 \mathrm{c}$ and d).

Particle density influences gravitational settling and the processes related to particle inertia, such as impaction. We take particle density from 1000 to $5000 \mathrm{~kg} \mathrm{~m}^{-3}$ to extend the application of the scheme to some special aerosol, such as heavy metal aerosol. As shown in Fig. 5a, particle deposition is not very sensitive to particle density, because the variability of particle density only slightly affects the deposition of particles larger than $5 \mu \mathrm{m}$ via the modification of gravitational settling.

We now examine the sensitivity of the scheme to aerodynamic parameters. Friction velocity is an aerodynamic parameter which influences the entire deposition process from turbulent diffusion to surface collection. As shown in Fig. 5b, the influence of $u_{*}$ is predominant for particles smaller than $10 \mu \mathrm{m}$, for which the deposition depends strongly on turbulent diffusion. An increased friction velocity also improves the surface collection due to impaction and interception and hence results in a noticeable enhancement of deposition for particles between 0.1 and $10 \mu \mathrm{m}$.

Then, we consider the sensitivity of the scheme to surface characteristics. Roughness element size affects the surfacecollection efficiency and two parameters are used to describe the surface characteristics in the new scheme. One is roughness element diameter, $d_{\mathrm{c}}$, and the other the micro-roughness parameter, $A_{\text {in }}$. Micro-roughness features, such as hair on the roughness element, enhance the surface-collection efficiency due to interception (Chamberlain, 1967; S82). For a certain micro-roughness feature ( $A_{\text {in }}=$ constant), the influence of $d_{\mathrm{c}}$ can be readily analyzed. As Fig. $5 \mathrm{c}$ shows, $d_{\mathrm{c}}$ mainly affects the deposition of particles in the size range of 0.01 to $10 \mu \mathrm{m}$, because it determines the collection efficiency due to impaction and interception. For particles with size in this range, deposition velocity is increased with decreasing $d_{\mathrm{c}}$ because of the improved interception. For particles from 10 to $50 \mu \mathrm{m}$, impaction slightly increases with roughness element size and so does deposition velocity.

While $d_{\mathrm{c}}$ is usually too large to affect interception, the influence of $A_{\text {in }}$ is significant and most profound on the deposition of particles in the size range of 0.1 to $10 \mu \mathrm{m}$ (Fig. 5d).

The parameter $R$ describes the rebound probability when a particle collides with the surface and particle rebound may reduce surface collection effect. As shown in Fig. 5e, the influence of $R$ on particle deposition is not obvious. Only 

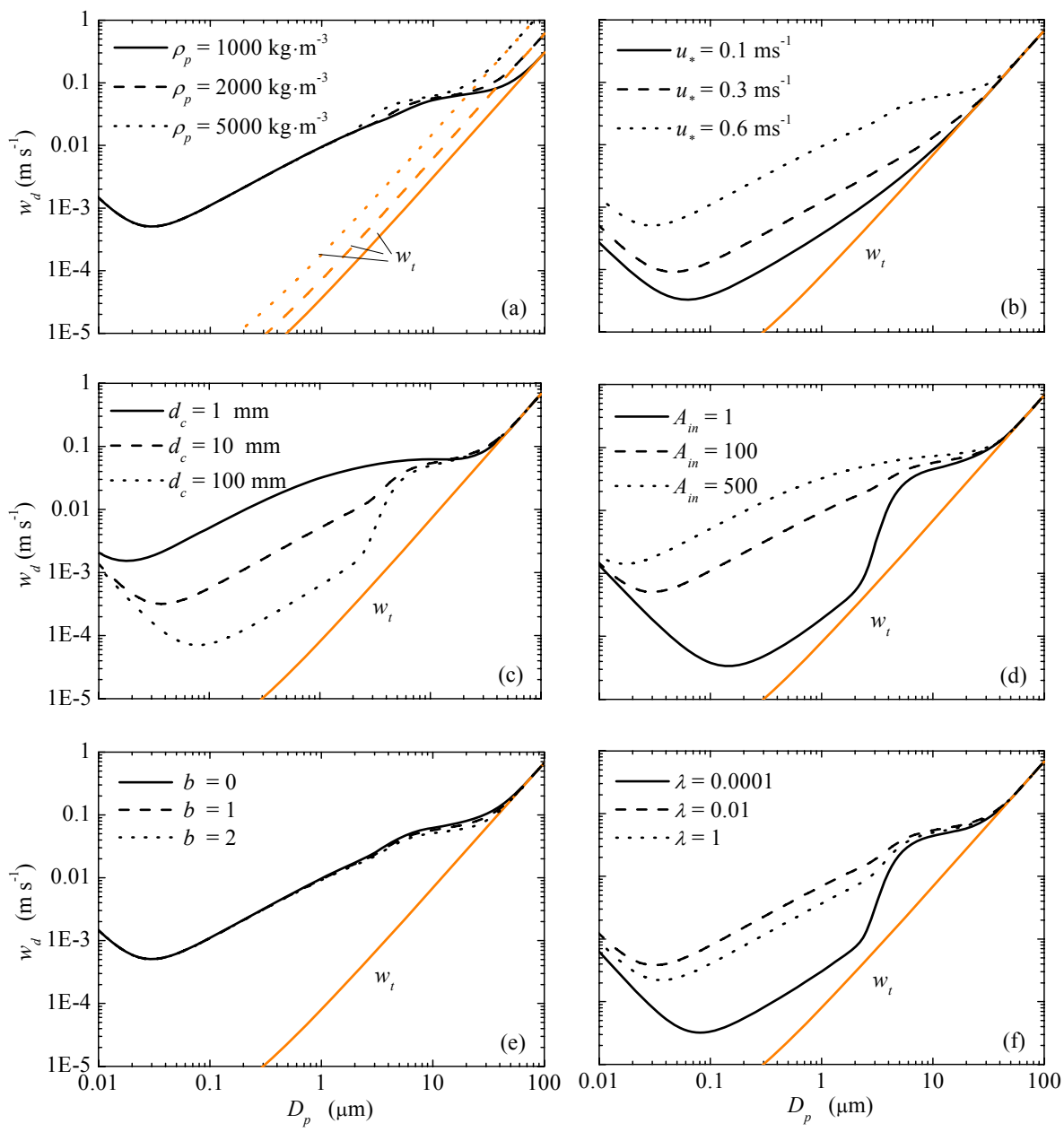

Figure 5. Sensitivity of deposition velocity to (a) particle density, (b) friction velocity, (c) roughness element size, (d) surface microroughness, (e) rebound probability and (f) element frontal area index. The deposition velocity is calculated for the reference height $1 \mathrm{~m}$ and the relevant parameter is evaluated as follows unless otherwise stated: $\rho_{\mathrm{p}}=2200 \mathrm{~kg} \mathrm{~m}^{-3}, u_{*}=0.6 \mathrm{~m} \mathrm{~s}^{-1}, z_{0}=10 \mathrm{~mm}, \mathrm{z}_{\mathrm{d}}=100 \mathrm{~mm}$, $h_{\mathrm{c}}=150 \mathrm{~mm}, d_{\mathrm{c}}=5 \mathrm{~mm}, A_{\text {in }}=100, b=1$ and $\lambda=0.1$.

for coarse particles larger than $5 \mu \mathrm{m}$, the deposition velocity slightly decreases with increasing $R$.

Roughness element frontal area index is a parameter used to describe the element distribution on the surface, which is also employed in the drag partition theory. We now test its influence on particle deposition. As shown in Fig. 5f, deposition velocity first increases, then decreases with increasing frontal area index. The influence is apparent for particles smaller than $50 \mu \mathrm{m}$, especially for particles in the range of 0.1 to $1 \mu \mathrm{m}$. Figure $5 \mathrm{f}$ suggests that in the case of a small frontal area index, the roughness elements make the surface rougher and enhance the surface collection, but as the number of roughness elements further increases, the surface becomes again smoother and the surface collection efficiency is decreased.

\section{Summary and discussion}

A new particle deposition scheme is proposed by adequate treatment of the gravitational settling effect and by taking into account the impact of roughness elements on turbulent diffusion and surface collection. The relationship between the aerodynamics and surface collection process is established, and the effect of the roughness elements on particle deposition is incorporated in the scheme by using the analogy of deposition flux partition to drag partition. Also, a modified expression for interception is proposed to account for the micro-roughness effect of the elements.

The new scheme has been tested against the wind-tunnel experimental data and good agreement between the scheme predictions and the observations is achieved. A new and more realistic explanation based on the new scheme is proposed for the enhanced particle deposition over water surfaces, i.e., water surface under windy conditions should be treated as 
a rough surface due to waves and spray droplets. We have however not yet validated the scheme against field observations. As wind-tunnel data have limitations due to simple turbulence and simple surface conditions, we cannot claim that the scheme is sufficiently and thoroughly tested. Also, we do not claim that our scheme is superior to the existing schemes, such as those of Zhang et al. (2001), Petroff and Zhang (2010), Kouznetsov and Sofiev (2012) etc. It appears desirable to do a thorough comparison with the other existing schemes, together with the other model developers, against a reliable field data set.

The sensitivity of the new scheme to some of the important input parameters has been tested. It is found that particle density and particle rebound probability mostly influence the deposition of coarse particles larger than $5 \mu \mathrm{m}$; the size and micro-roughness characteristics of the roughness elements influence interception noticeably and hence the deposition of particles in the size range of 0.1 to $10 \mu \mathrm{m}$; friction velocity affects the entire deposition process and significantly influences the deposition of particles smaller than $10 \mu \mathrm{m}$; roughness element frontal area index has a predominant effect on surface collection efficiency and influences the deposition of particles smaller than $50 \mu \mathrm{m}$.

While we believe the new scheme has improved the capacity for parameterizing particle deposition over rough surfaces, some questions remain unanswered and future research is required in the following areas.
This section deals with the effect of wind intermittency. In our study, we assumed the wind is steady and the effect of wind intermittency is neglected. But wind intermittency may have a significant effect on particle deposition, including particle transport in the upper layer and particle collection in the lower layer (Fig. 3). While some studies on the topic already exist, e.g., the treatment of the effect of wind intermittency on aerodynamic resistance by Zhang et al. (2001) and Seinfeld and Pandis (2006), the influence of wind intermittency on the particle collection process deserves further research.

Deposition on complex surfaces: only surfaces with relatively simple and uniform elements are tested in our study, but natural surfaces are much more complex. For example, how to predict particle deposition to surfaces with multi-size roughness elements is important for regional and global particle models.

Effect of roughness element interaction on element collection efficiency: in analogy to the drag partition theory, an expression for describing the distribution of total deposited particle on different parts of the surface (side area of the roughness elements or upward facing surface) has been proposed in our study. But the collection efficiency of roughness element is evaluated based on the study of isolated obstacle. The effect on element collection efficiency due to the interactions between the roughness elements remains rather unclear. 


\section{Appendix A}

Table A1. List of symbols.

\begin{tabular}{|c|c|c|}
\hline$A_{\text {in }}$ & Empirical parameter for surface micro-roughness characteristics & / \\
\hline$B_{1}, B_{2}$ & Empirical constant & / \\
\hline$b$ & Numerical constant in rebound expression & / \\
\hline$c$ & Particle concentration & $\mathrm{kg} \mathrm{m}^{-3}$ \\
\hline$C_{\mathrm{c}}$ & Cunningham correction factor & / \\
\hline$C_{\mathrm{d}}$ & Drag coefficient for obstacle & / \\
\hline$D_{\mathrm{p}}, D_{\mathrm{p}, \delta}$ & Dry/wet particle diameter & $\mathrm{m}$ \\
\hline$d_{\mathrm{c}}, d_{\mathrm{c}}^{1}, d_{\mathrm{c}}^{\mathrm{s}}$ & $\begin{array}{l}\text { Dimension of the roughness elements, large collector } \\
\text { (i.e., roughness elements) and small collector }\end{array}$ & $\mathrm{m}$ \\
\hline$E, E^{\mathrm{B}}, E^{\mathrm{in}}, E^{\mathrm{im}}$ & Element collection efficiency for different mechanisms & l \\
\hline$F_{\mathrm{d}}$ & Particle deposition flux & $\mathrm{kg} \mathrm{m}^{-2} \mathrm{~s}^{-1}$ \\
\hline$g$ & Gravitational acceleration & $\mathrm{m} \mathrm{s}^{-2}$ \\
\hline$h$ & Thickness of surface collection layer & $\mathrm{m}$ \\
\hline$h_{\mathrm{c}}$ & Height of roughness element & $\mathrm{m}$ \\
\hline$K_{\mathrm{B}}$ & Boltzmann constant & $\mathrm{JK}^{-1}$ \\
\hline$K_{\mathrm{p}}$ & Particle eddy diffusivity & $\mathrm{m}^{2} \mathrm{~s}^{-1}$ \\
\hline$K_{\mathrm{T}}$ & Turbulent (or eddy) viscosity & $\mathrm{m}^{2} \mathrm{~s}^{-1}$ \\
\hline$k_{\mathrm{p}}$ & Brownian diffusion coefficient & $\mathrm{m}^{2} \mathrm{~s}^{-1}$ \\
\hline$R$ & Reduction in collection caused by rebound & l \\
\hline $\operatorname{Re}$ & Reynolds number & / \\
\hline$r_{\mathrm{a}}$ & Aerodynamic resistance & $\mathrm{s} \mathrm{m}^{-1}$ \\
\hline$r_{\mathrm{s}}$ & Surface collection resistance & $\mathrm{s} \mathrm{m}^{-1}$ \\
\hline$r_{\mathrm{g}}$ & Resistance of gravity (inverse of terminal velocity) & $\mathrm{s} \mathrm{m}^{-1}$ \\
\hline Sc & Schmidt number & l \\
\hline$S c_{\mathrm{T}}$ & Turbulent Schmidt number $K_{\mathrm{T}} / K_{\mathrm{p}}$ & l \\
\hline$S t$ & Stokes number & l \\
\hline$T_{\mathrm{p}}$ & Relaxation time of particle & $\mathrm{s}$ \\
\hline$T_{\mathrm{p}}^{+}$ & Dimensionless particle relaxation time & l \\
\hline$u_{\mathrm{a}}$ & Horizontal velocity of air & $\mathrm{ms}^{-1}$ \\
\hline$u_{*}$ & Friction velocity & $\mathrm{m} \mathrm{s}^{-1}$ \\
\hline$w_{\mathrm{d}}$ & Deposition velocity $w_{\mathrm{d}}=-F_{\mathrm{d}} / C$ & $\mathrm{~m} \mathrm{~s}^{-1}$ \\
\hline$w_{\mathrm{t}}$ & Terminal velocity & $\mathrm{m} \mathrm{s}^{-1}$ \\
\hline$z, z_{\mathrm{r}}$ & Height and reference height & $\mathrm{m}$ \\
\hline$z_{0}, z_{\mathrm{d}}$ & Roughness length and zero-plane displacement & $\mathrm{m}$ \\
\hline \multicolumn{3}{|l|}{ Greek symbols } \\
\hline$\beta$ & $\begin{array}{l}\text { Ratio of the drag coefficient for isolated roughness element } \\
\text { to that of bare surface, evaluated to } 200 \text { in this study. }\end{array}$ & I \\
\hline$\delta$ & Thickness of laminar layer & $\mathrm{m}$ \\
\hline$\eta$ & Basal area index & / \\
\hline$\kappa$ & von Karman constant & / \\
\hline$\lambda$ & Frontal area index & I \\
\hline$\mu$ & Dynamic viscosity of air & $\mathrm{kg} \mathrm{m}^{-1} \mathrm{~s}^{-1}$ \\
\hline$v$ & Kinematic viscosity of air & $\mathrm{m}^{2} \mathrm{~s}^{-1}$ \\
\hline$\rho_{\mathrm{p}}, \rho_{\mathrm{a}}$ & Particle/air density & $\mathrm{kg} \mathrm{m}^{-3}$ \\
\hline$\tau, \tau_{\mathrm{c}}, \tau_{\mathrm{s}}, \tau_{\mathrm{r}}$ & Drag exerted on different parts of the surface & $\mathrm{Nm}^{-2}$ \\
\hline
\end{tabular}


Acknowledgements. This work is supported by the DFG (Deutsche Forschungsgemeinschaft) project "A Wind-tunnel Study on Dustdeposition Mechanics and Validation of Dust-deposition Schemes", Natural Science Foundation of China (Project 11172118) and the Innovative Research Groups of the National Natural Science Foundation of China (Project 11121202).

Edited by: X. Liu

\section{References}

Arya, S. P. S.: A drag partition theory for determining the largescale roughness parameter and wind stress on the Arctic pack ice, J. Geophys. Res., 80, 3447-3454, 1975.

Chamberlain, A. C.: Transport of Lycopodium spores and other small particles to rough surfaces, Proc. Roy. Soc. Lnd. A, 296, 45-70, 1967.

Csanady, G. T.: Turbulent diffusion of heavy particles in the atmosphere, J. Atmos. Sci., 20, 201-208, 1963.

Droppo, J. G.: Improved Formulations for Air-Surface Exchanges Related to National Security Needs: Dry Deposition Models, Pacific Northwest National Laboratory, Richland, WA, 2006.

Fitzgerald, J. W.: Approximation Formulas for the Equilibrium Size of an Aerosol Particle as a Function of Its Dry Size and Composition and the Ambient Relative Humidity, J. Appl. Meteorol., 14, 1044-1049, 1975.

Fuchs, N. A.: Mechanics of Aerosols, Pergamon, New York, 135-142, 1964.

Gerber, H. E.: Relative-humidity parameterization of the Navy Aerosol Model (NAM) (No. NRL-8956), Naval Research Lab, Washington DC, 1985.

Giorgi, F.: Dry deposition velocities of atmospheric aerosols as inferred by applying a particle dry deposition parameterization to a general circulation model, Tellus B, 40, 23-41, 1988.

Hicks, B. B., Baldocchi, D. D., Meyers, T. P., Hosker Jr, R. P., \& Matt, D. R.: A preliminary multiple resistance routine for deriving dry deposition velocities from measured quantities, Water Air Soil Poll., 36, 311-330, 1987.

Kouznetsov R. and Sofiev M.: A methodology for evaluation of vertical dispersion and dry deposition of atmospheric aerosols, J. Geophys. Res., 117, D01202, doi:10.1029/2011JD016366, 2012.
Petroff, A. and Zhang, L.: Development and validation of a size-resolved particle dry deposition scheme for application in aerosol transport models, Geosci. Model Dev., 3, 753-769, doi:10.5194/gmd-3-753-2010, 2010.

Petroff, A., Mailliat, A., Amielh, M., Anselmet, F.: Aerosol dry deposition on vegetative canopies. Part II: A new modelling approach and applications, Atmos. Environ., 42, 3654-3683, 2008.

Raupach, M.: Drag and drag partition on rough surfaces, Bound.Lay. Meteorol., 60, 375-395, 1992.

Raupach, M. R., Briggs, P. R., Ford, P. W., Ahmad, N., and Edge, V. E.: Endosulfan transport: II. Modeling airborne dispersal and deposition by spray and vapor, J. Environ. Qual., 30, 729-740, 2001.

Sehmel, G. A.: Particle and gas dry deposition: A review, Atmos. Environ., 14, 983-1011, 1980.

Seinfeld, J. H. and Pandis, S. N.: Atmospheric chemistry and physics: from air pollution to climate change, second edition, John Wiley \& Sons, Inc., Hoboken, 900-929, 2006.

Shao, Y.: Physics and modelling of wind erosion, second edition, Springer-Verlag, Berlin, 277-288, 2008.

Shao, Y. and Yang, Y.: A scheme for drag partition over rough surfaces, Atmos. Environ., 39, 7351-7361, 2005.

Shao, Y. and Yang, Y.: A theory of drag partition over rough surfaces, J. Geophys. Res., 113, F02S05, doi:10.1029/2007JF000791, 2008.

Slinn, S. A. and Slinn, W. G. N.: Predictions for particle deposition on natural waters, Atmos. Environ., 14, 1013-1016, 1980.

Slinn, W. G. N.: Predictions for particle deposition to vegetative canopies, Atmos. Environ., 16, 1785-1794, 1982.

Venkatram, A. and Pleim, J.: The electrical analogy does not apply to modeling dry deposition of particles, Atmos. Environ., 33, 3075-3076, 1999.

Wesely, M. L. and Hicks, B. B.: A review of the current status of knowledge on dry deposition, Atmos. Environ., 34, 2261-2282, 2000.

Zhang, J., Shao, Y., and Huang, N.: Measurements of dust deposition velocity in a wind-tunnel experiment, Atmos. Chem. Phys., 14, 8869-8882, doi:10.5194/acp-14-8869-2014, 2014.

Zhang, L., Gong, S., Padro, J., and Barrie, L.: A size-segregated particle dry deposition scheme for an atmospheric aerosol module, Atmos. Environ., 35, 549-560, 2001. 